\title{
Several types of soft tissue sarcomas originate from the malignant transformation of adipose tissue-derived stem cells
}

\author{
HUI CHEN ${ }^{1,2}$, SONG ZHANG ${ }^{1}$, JIAN-CHENG WEN ${ }^{1}$, JIA-KUN ZHENG ${ }^{1}$, QIANG CHEN ${ }^{1}$, WEN-YU LI ${ }^{1}$, \\ PENG-PENG WANG ${ }^{1,3}$, LIAN MA ${ }^{3}$, TIAN-HUA HUANG ${ }^{2}$, GE HUANG ${ }^{2}$ and LI-YE YANG ${ }^{1,2}$ \\ ${ }^{1}$ Central Laboratory, Chaozhou Central Hospital, Chaozhou 521021; ${ }^{2}$ Medical College of Shantou University, Shantou 515041; \\ ${ }^{3}$ Department of Pediatrics, The Second Affiliated Hospital of the Medical College of Shantou University, \\ Shantou 515031, P.R. China
}

Received November 12, 2009; Accepted February 5, 2010

DOI: $10.3892 / \mathrm{mmr} 00000277$

\begin{abstract}
The cellular origin of soft tissue sarcomas (STSs) is not fully understood. The cancer stem cell hypothesis presumes that tumors originate from the malignant transformation of stem cells. As a type of multipotent stem cell, adipose tissue-derived stromal/stem cells (ADSCs), which possess an unexpected degree of plasticity and often reside in other tissues, may represent a potential source of soft tissue sarcoma. To ascertain whether ADSCs are responsible for the formation of STSs, ADSCs from mice were cultured and treated with 3-methycholanthrene to derive transformed cells. These transformed ADSCs were then injected subcutaneously into immunodeficient mice to test their tumorigenic potential. We found that they generated several types of STSs, including synovial sarcoma, malignant fibrous histiocytoma and fibrosarcoma. This is the first study to report that ADSCs may be the potential initiating cells for synovial sarcoma. Our findings indicate that STSs might originate from malignantly transformed ADSCs.
\end{abstract}

\section{Introduction}

The cancer stem cell hypothesis has gained significant recognition as the descriptor of tumorigenesis and the cellular origin of tumors. It is well known that multipotent adult progenitor/ stem cells can differentiate into diverse cellular lineages and can be recruited to areas of profound tissue injury, while in some settings they may also initiate malignant transformation. Therefore, it is believed that tumors might originate from the malignant transformation of their tissue-specific stem cells $(1,2)$. The discovery of multipotent progenitor/stem cells with the capacity for self-renewal raises the possibility that cancer

Correspondence to: Dr Li-Ye Yang, Central Laboratory, Chaozhou Central Hospital, No. 84 Huan-Cheng-Xi Road, Chaozhou 521021, Guangdong Province, P.R. China

E-mail: yangleeyee@sina.com

Key words: soft tissue sarcoma, stem cell, adipose tissue-derived stem cells, malignant transformation, synovial sarcoma stem cells arise from the stem cells of other tissues and initiate other cancer types, including solid cancers (3). There was no experimental evidence regarding the contribution of tissue stem cells other than the tissue of origin to tumor formation until Houghton et al (4) found that gastric cancers originate from bone marrow-derived sources, proposing mesenchymal stem cells (MSCs) as the most likely candidate. Recent studies have also indicated that malignantly transformed MSCs give rise to different types of tumors in vivo (5-7). In addition, as a type of multipotent adult progenitor/stem cell, adipose tissue-derived stromal/stem cells (ADSCs), which possess an unexpected degree of plasticity and often reside in other tissues $(8,9)$, may represent potential initiating cells of malignancy, including soft tissue sarcomas (STSs). ADSCs harvested from adipose tissue are a type of multipotent progenitor/stem cell that were found to have an equal potential to differentiate into cells and tissues of mesodermal origin, such as adipocytes, cartilage, bone, skeletal muscle and tendons, when cultivated under lineage-specific conditions $(10,11)$.

STSs, which constitute less than $1 \%$ of adult solid tumors, are considered to be the most aggressive form of malignancy. Research has indicated that STSs are putative mesenchymalderived tumors arising predominantly from the embryonic mesoderm (12-14). However, to date, the specific tumor-initiating cells of STSs remain unknown. A considerable volume of recent evidence indicates that transformed bone marrow mesenchymal stem cells (BMMSCs) produce tumors, including carcinoma and sarcoma, and may be responsible for the development of STSs (7,14-18). Due to the similar biological characteristics of ADSCs and BMMSCs, we considered that ADSCs are potentially more likely be the specific tumor-initiating cells of STSs than BMMSCs. To demonstrate this hypothesis, we treated ADSCs with 3-methycholanthrene (MCA), a potent carcinogen, to derive transformed ADSCs, then studied the characteristics of the transformed ADSCs in vitro and in vivo.

\section{Materials and methods}

Isolation and culture of ADSCs from mice. Subperitoneal adipose tissues were cut from the abdomens of 3- to 6-week-old Balb/C mice (Experimental Animal Centre of Sun Yat-Sen University, Guangzhou, P.R. China) under sterile 
Table I. Sequence of the PCR primers.

Gene

p53 exon 5-6

p53 exon 7

p53 exon 8-9

$\beta$-actin
Primer sequence

Product size (bp)

F: 5'-CCTTGACACCTGATCGTTACTCG-3'

507

R: 5'-AGAAAGTCAACATCAGTCTAGGC-3'

F: 5'-TGTGCCGAACAGGTGGAATATCC-3'

322

R: 5'-ACTCGTGGAACAG AAACAGGCAG-3'

F: 5'-GGCCTAGTTTACACACAGTCAGG-3'

495

R: 5'-CACGGCTAGAGATAAAGCCACTG-3'

F: 5'-TGGCACCACACCTTCTACAATGAGC-3'

396

R: 5'-GCACAGCTTCTCCTTAATGTCACGC-3'

conditions and placed in phosphate-buffered saline (PBS). Blood vessels and fibrous materials were cut off and discarded. After extensive washing with PBS, the tissue samples were cut into small pieces and digested using a modification of the method described by Zuk et al $(10,11)$. The samples were then incubated with intermittent agitation for 45-60 min in calcium- and magnesium-free PBS, pH 7.4, containing $0.75 \mathrm{mg} / \mathrm{ml}$ collagenase. After dispersion, the cells were filtered through a $250-\mu \mathrm{m}$ mesh and centrifuged at $1,000 \mathrm{x} \mathrm{g}$ for $10 \mathrm{~min}$ at room temperature. Collagenase digestion of adipose tissue resulted in the formation of two distinct fractions: floating mature adipose cells and sedimented stromal-vascular cells. These latter were the source of the ADSCs. After the floating fat cells were removed by aspiration, the sedimented cells were resuspended in $160 \mathrm{mM}$ $\mathrm{NH}_{4} \mathrm{Cl}$ and incubated for $10 \mathrm{~min}$ at room temperature to lyse contaminating red blood cells. Centrifugation was repeated as above, and the resulting solution was filtered through a $100-\mu \mathrm{m}$ metal mesh to remove cellular debris, then transferred to a $75 \mathrm{~cm}^{2}$ flask (Corning) containing medium and incubated in an atmosphere of $5 \% \mathrm{CO}_{2} / 95 \%$ air at $37^{\circ} \mathrm{C}$. Following the first $24 \mathrm{~h}$ of incubation, the cultures were washed with PBS to remove non-adherent and retaining adherent cells as ADSCs. The cells were selected by their plastic adherence and rapid proliferation. ADSCs were cultured in Dulbecco's modifed Eagle's medium (DMEM; Gibco) containing 10\% FCS (Lanzhou Minhai Bio. Co., P.R. China), $2 \mathrm{mM}$ glutamine, $100 \mathrm{U} / \mathrm{ml}$ penicillin and $100 \mu \mathrm{g} / \mathrm{ml}$ streptomycin (Gibco). Upon achieving $85 \%$ confluence, the cells were treated with trypsin $\left(0.25 \%\right.$ in $5 \mathrm{mM}$ EDTA) for $5 \mathrm{~min}$ at $37^{\circ} \mathrm{C}$, washed with culture medium, sedimented $(600 \mathrm{x} \mathrm{g})$ for $10 \mathrm{~min}$ at room temperature, and finally plated into culture plates or flasks for further culture.

Induction of ADSCs with MCA. Primary ADSCs were cultured for 10-14 days in the above-mentioned medium. Upon achieving 80-90\% confluence, they were passaged and randomly divided into two groups. The first group of ADSCs was treated with $1 \mu \mathrm{g} / \mathrm{ml}$ MCA (Sigma) in culture medium for 1 week, then continually cultured in medium without MCA. MCA was dissolved in dimethyl sulfoxide (DMSO; Sigma) to yield a stock solution of $200 \mu \mathrm{g} / \mathrm{ml}$, which was stored in the dark at $4^{\circ} \mathrm{C}(15)$ then added to the medium for a final concentration of DMSO of $0.5 \%$. The second group was treated with
$0.5 \%$ DMSO as the control. In order to verify whether ADSCs treated with MCA were transformed, morphological changes were observed daily under an inverted microscope.

Isolation of a single clone. Transformed ADSCs were serially diluted into expansion medium in 96-well plates so that only a single clone was expanded. Once the single cell in a well had multiplied and achieved $90 \%$ confluence, the cells were transferred to culture flasks for propagation.

Immunocytochemistry of transformed ADSCs. Transformed ADSCs were cultured in 3.5-cm dishes. Upon achieving $80-90 \%$ confluence, they were fixed with $4 \%$ paraformaldehyde for $15 \mathrm{~min}$, followed by washing three times for $2 \mathrm{~min}$ each with PBS. Endogenous peroxidase activity was quenched in $3 \% \mathrm{H}_{2} \mathrm{O}_{2}$ for $15 \mathrm{~min}$. The cells were then permeated in $0.25 \%$ Triton X-100 for $15 \mathrm{~min}$ with primary antibodies (Beijing Zhongshan Jinqiao Co., P.R. China) including pan-cytokeratin (pan-CK) (1:1), proliferating cell nuclear antigen (PCNA) (1:1), blood vessel endothelial growth factor-c (VEGF-c) (1:1) and vimentin (1:50), then incubated in a humidity chamber at room temperature for $2 \mathrm{~h}$. A broad spectrum secondary antibody staining kit was used (SP-9000; Beijing Zhongshan Jinqiao Co.) with 3-amino-9-ethyl carbazole (AEC; Beijing Zhongshan Jinqiao Co.) as the chromagen. The samples were finally observed under a high-power optical microscope.

Chromosome preparation and analysis. In order to get the message of the chromosomes to the transformed cells, chromosome preparation was carried out following routine methods and procedures (19). Once the procedures had been completed, the slide samples were harvested and observed under a microscope.

DNA amplification and sequencing of the p53 gene. Genomic DNA was isolated from transformed ADSCs using a genomic DNA isolation kit [Universal Genomic DNA Extraction kit ver. 3.0, Takara Biotechnology (Dalian) Co. Ltd.] according to the manufacturer's instructions. The primers for PCR were synthesized at the Shanghai Sangon Biological Engineering Technology \& Services Co. Ltd. PCR was performed for exons 5-6, 7, and 8-9 of p53 in a KP-TC48 thermal cycler (Hybribio Ltd.). $\beta$-actin primers were used as a positive control for the presence of DNA. Primer sequences are listed in Table I. 

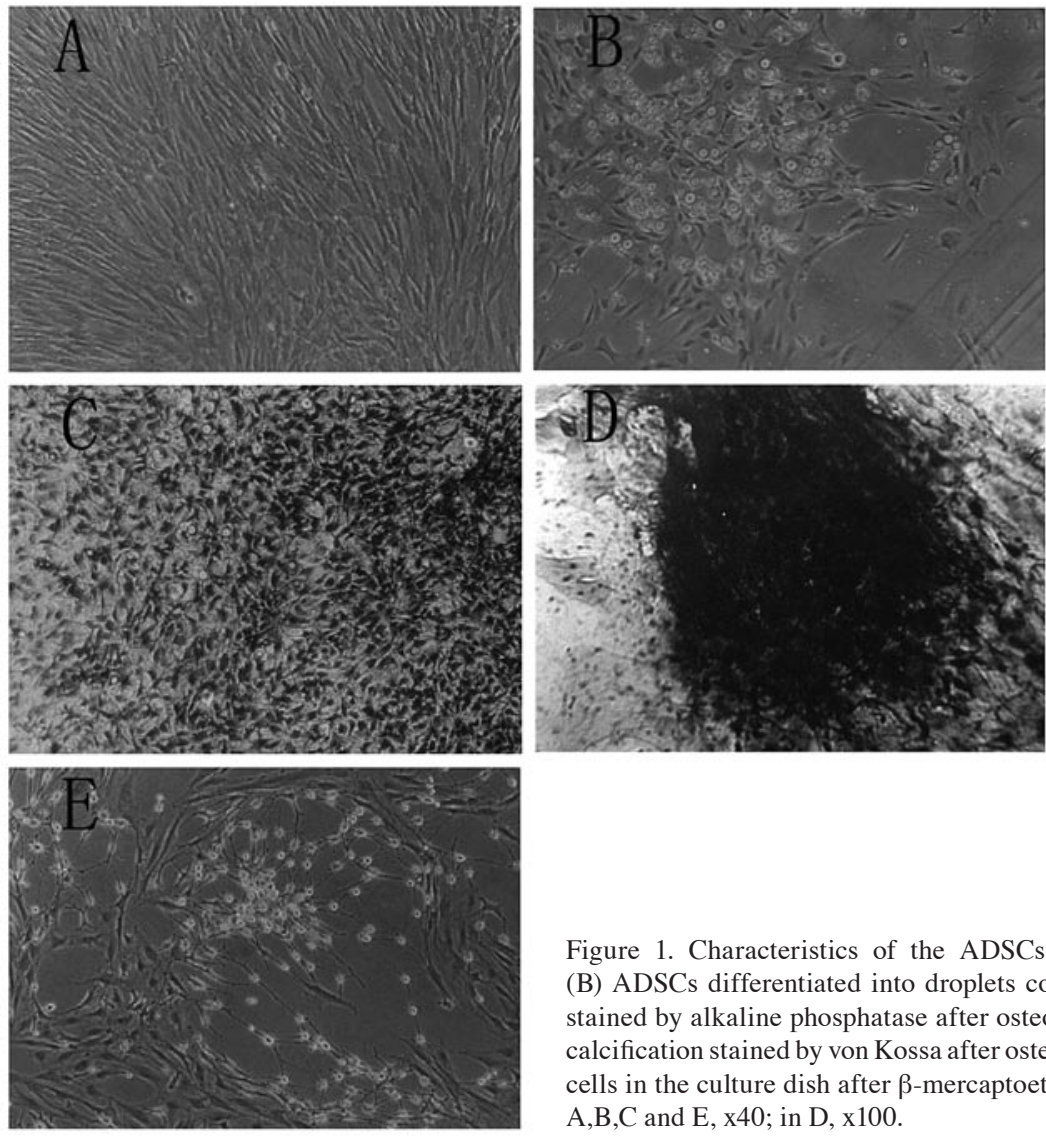

Figure 1. Characteristics of the ADSCs. (A) Primary cultured ADSCs. (B) ADSCs differentiated into droplets containing adipocytes. (C) ADSCs stained by alkaline phosphatase after osteogenic induction. (D) A nodule of calcification stained by von Kossa after osteogenic induction. (E) Neuron-like cells in the culture dish after $\beta$-mercaptoethanol induction. Magnification in $\mathrm{A}, \mathrm{B}, \mathrm{C}$ and $\mathrm{E}, \mathrm{x} 40$; in $\mathrm{D}, \mathrm{x} 100$.

Fragments were individually amplified in PCR reaction mixtures $(50 \mu \mathrm{l})$ containing $10 \mathrm{mM}$ Tris $(\mathrm{pH} \mathrm{8.6)}, 50 \mathrm{mM} \mathrm{KCl}$, $1.5 \mathrm{mM} \mathrm{MgCl}_{2}, 0.4 \%$ DMSO, $0.2 \mathrm{mM}$ dNTPs, $0.4 \mathrm{mM}$ of each primer, 50 ng of genomic DNA and 5 units of Taq polymerase (Takara Taq ${ }^{\mathrm{TM}}$, Takara Biotechnology Co. Ltd.). Cycling conditions were as follows: an initial $3 \mathrm{~min}$ at $95^{\circ} \mathrm{C}$, followed by 33 cycles $\left(95^{\circ} \mathrm{C}, 1 \mathrm{~min} / 55^{\circ} \mathrm{C}, 1 \mathrm{~min} / 72^{\circ} \mathrm{C}, 1 \mathrm{~min}\right)$ and a final 5 -min step at $72^{\circ} \mathrm{C}$. Amplified fragments were visualized on a $1.5 \%$ agarose gel stained with ethidium bromide and photographed. The PCR products of p53 exons 5-9 were sequenced directly by Shanghai Sangon Biological Engineering Technology \& Services Co. Ltd. using an ABI PRISM 3730 sequencer with BigDye terminator reagent. All samples were tested by simultaneous bi-directional sequencing.

Tumorigenesis in vivo. Approximately $4-5 \times 10^{6}$ transformed ADSCs or normal ADSCs were injected subcutaneously into 4-week-old immunodeficient Balb/C mice. Equal amounts of cells from each of 8 monoclonal lines were transplated into mice (Experimental Animal Centre of Guangzhou, University of Chinese Traditional Medicine, Guangzhou, P.R. China). Some mice were injected with control ADSCs, pre-treated with DMSO only.

Tissue collection and histological analysis. Most tumors appeared in the mice at the site of injection within 8 weeks, and the mice were sacrificed at 12 weeks after injection. The tumors were removed and fixed in 10\% neutral-buffered formalin for $24 \mathrm{~h}$ and embedded in paraffin for analysis. Tumors were sectioned $(4 \mu \mathrm{m})$ and stained using routine $\mathrm{H} \& \mathrm{E}$.
In order to identify the tumor types, histology was performed in combination with various immunostaining procedures. Immunochemistry was carried out using standard protocol. Primary antibodies (Beijing Zhongshan Jinqiao Co.) included the following: actin (1:1), CD34 (1:1), CD68 (1:1), pan-CK $(1: 1)$, desmin (1:50), epithelial membrane antigen (EMA) (1:1), lysozyme (1:1), PCNA (1:1), smooth muscle actin (SMA) $(1: 1)$, S-100 (1:1) and vimentin (1:50). Immunodetection was performed using the SP-9000 kit. The counterstain of preference for nuclear details was hematoxylin.

\section{Results}

ADSC morphology and stem cell characteristics. ADSCs were obtained from the abdomens of 3- to 6-week-old mice and were isolated based on their plastic adherence. Hematopoietic stem cells (HSCs) and other non-adherent cells were removed with changes of the medium. Primary ADSCs displayed typical fibroblast-like morphology and were maintained for long-term culture in vitro $(10,11,20)$ (Fig. 1A), and were capable of differentiating into adipocytes (Fig. 1B) and osteogenic cells (Fig. 1C and D) under specific induction $(10,21)$. These cells were induced into neuron-like cells by $\beta$-mercaptoethanol (Fig. 1E). The induction protocol was the same as previously described (22), and the neuron-like cells expressed neuron phenotypes (data not shown). These experiments demonstrated that the cultured ADSCs possessed the characteristics of multipotent differentiation $(10,11)$. 


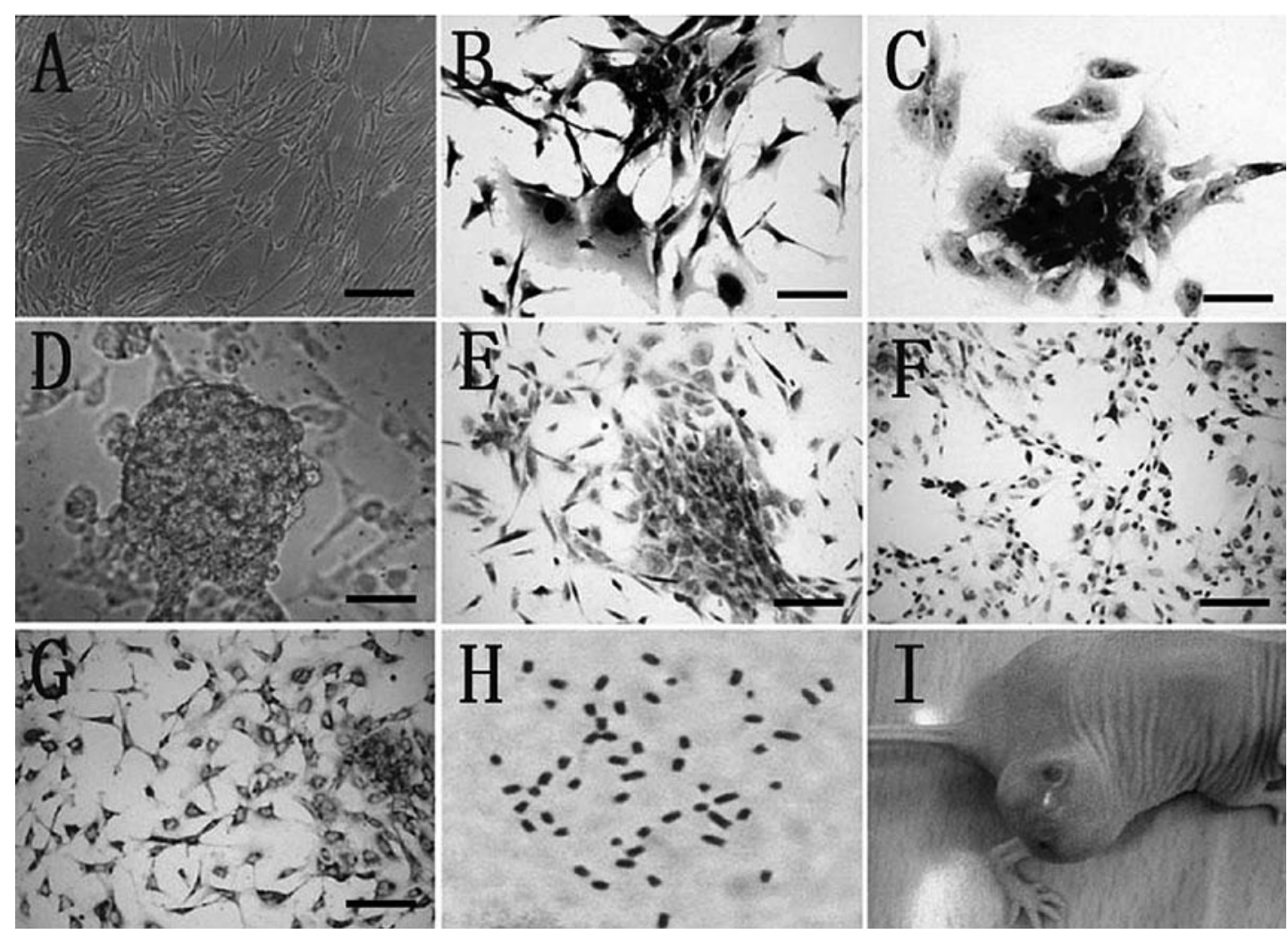

Figure 2. Characteristics of the transformed ADSCs. (A) Morphological characteristics of ADSCs before MCA treatment. (B) Morphological changes in the transformed ADSCs, cellular and nuclear atypia (stained with hematoxylin). (C) Increased number of nucleoli (stained with Giemsa). (D) Multicellular spheroids formed from the transformed ADSCs. Transformed ADSCs were positive for (E) PCNA, (F) VEGF-c and (G) vimentin. (H) Chromosome alterations in a transformed ADSC. (I) Tumor formation from transformed ADSCs. Bars: A, $100 \mu \mathrm{m}$; B, C and D, $50 \mu \mathrm{m}$; E, F and G, $70 \mu \mathrm{m}$.

A
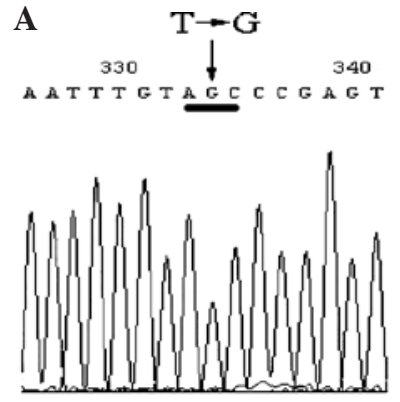

Figure 3. Mutation of p53. (A) The mutation site of exons 5-6 was NT 34907052 $(\mathrm{T} \rightarrow \mathrm{G})$. (B) The mutation site of exons 8-9 was NT $34908188(\mathrm{C} \rightarrow \mathrm{T})$

Malignant transformation of ADSCs. ADSCs treated as described previously with MCA were successively cultured for 4 months. A number of cells demonstrating the properties of malignant transformation appeared, showing morphological changes, rapid growth (almost without platform stage, with a cell doubling time of $\sim 24 \mathrm{~h}$ ), loss of contact inhibition and loss of polarity (Fig. 2B and C). Moreover, the transformed cells were tested for malignancy by examination of piled-up colonies (15), showing overlapped cells and sphere colony formation (Fig. 2D). Untransformed cells remained as monolayers. However, ADSCs treated only with DMSO entered the senescence phase after 2 months of culture (data not shown). These results were reproducible, as transformed ADSCs were derived in three independent experiments. We found that transformed cells possessed the characteristics of malignant cells, and that ADSCs treated with MCA underwent malignant transformation.

Cell markers for transformed ADSCs. During the routine passage of transformed cells, multiple cell shapes were frequently observed in the culture. We studied the phenotype of the transformed ADSCs by testing for tissue-specific markers. The cells stained positive for PCNA (Fig. 2E) and VEGF-c (Fig. 2F), the cell markers for cancer. They were also positive for vimentin (Fig. 2G) and SMA (data not shown), but negative for pan-CK, indicating a mesenchymal origin.

Aneuploidy in transfomed ADSCs. Metaphase chromosomes of transformed ADSCs were harvested as described previously and photographed under a microscope. Notably, 50 of the 50 cells analyzed were aneuploidy, with a total chromosome number ranging from 47 to 99 and hypertetraploid with a chromosome number between 81 and 99. These findings suggest that chromosomal alterations are common events in the progression of the malignant transformation of ADSCs. A representative metaphase showing a transformed ADSC with 52 chromosomes is shown in Fig. $2 \mathrm{H}$.

p53 gene mutation in transformed ADSCs. The PCR products of p53 exons 5-6, 7 and 8-9 were successfully amplified, and the mutations of these exons were further confirmed by direct sequencing. Sequenced p53 exons 5-6, 7 and 8-9 were compared to wt p53 in the Genebank. Gene mutations were identified; all were point mutations. The mutation site of 


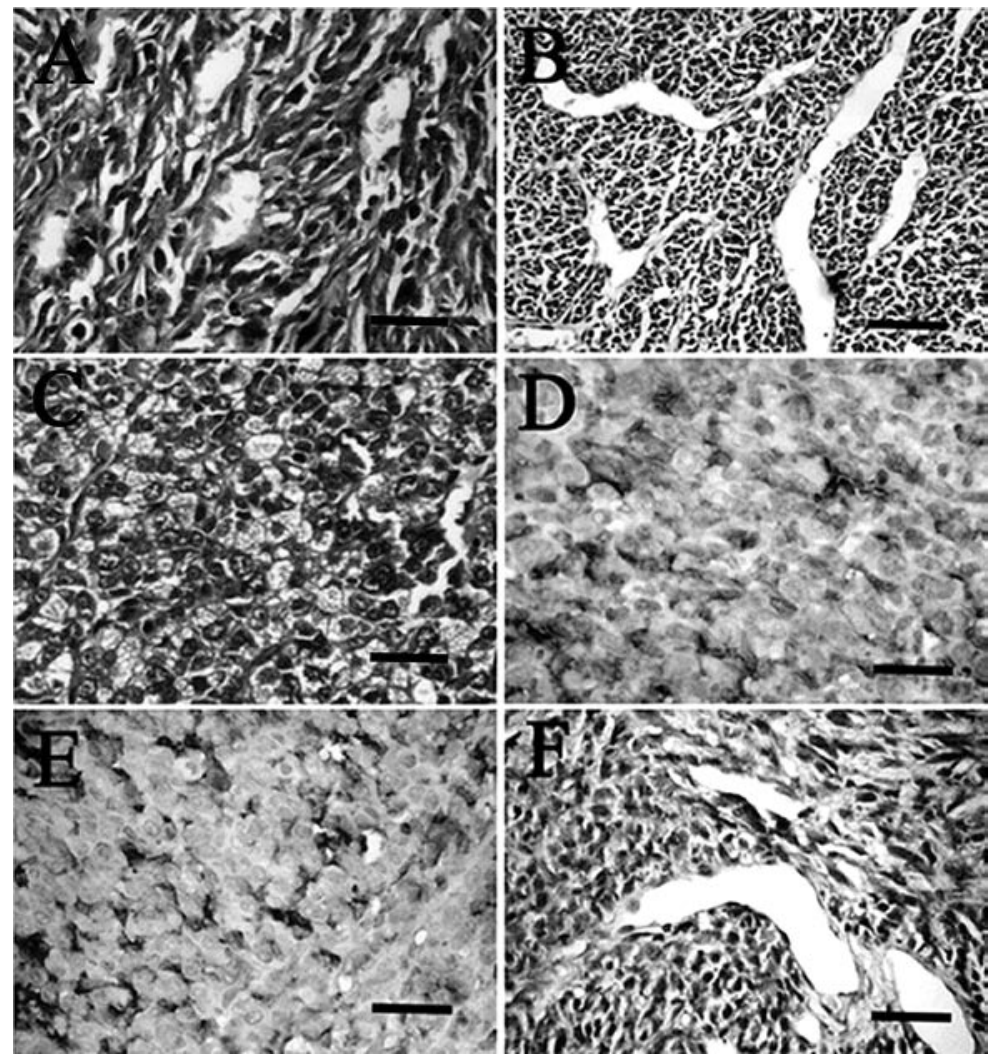

Figure 4. Images of H\&E staining and immunohistochemical analysis of the sections showed a synovial sarcoma tumor type (biphasic type). (A) Glandlike lacune structure. (B) A considerable number of hemangiopericytoma-like structures in poorly differentiated areas. (C) Adenocarcinoma-like structure. Immunohistochemical images for (D) pan-CK, (E) EMA and (F) vimentin were diffusely positive. Bars: A, C, D, E and F, $25 \mu \mathrm{m}$; B, $100 \mu \mathrm{m}$.

exon 5-6 was NT $34907052(\mathrm{~T} \rightarrow \mathrm{G})$ (Fig. 3A), the mutation of exon 7 was not found, and the mutation site of exon 8-9 was NT 34908189 (C $\rightarrow$ T) (Fig. 3B).

Transformed ADSCs produced STSs in vivo. Transformed cells were injected subcutaneously into immunodeficient mice and formed tumors in 16 of the 18 animals. Most tumors appeared at the site of injection within 6-10 weeks (Fig. 2I), whereas mice inoculated with ADSCs treated only with DMSO did not form tumors. Tumor tissues were analyzed by histology and immunohistochemistry to identify tumor type. Sixteen tumors displayed various sarcoma characteristics. Two of the 16 tumors exhibited a biphasic, spindle- and epithelial-appearing form and gland-like (Fig. 4A), hemangiopericytoma-like (Fig. 4B) and adenocarcinoma-like structures (Fig. 4C). Immunohistochemical results revealed that these tumors were locally positive for pan-CK (Fig. 3D), EMA (Fig. 4E) and vimentin (Fig. 4F). Results for S-100 protein, actin, CD34, CD68, desmin, lysozyme and SMA were negative (data not shown). These characteristics were consistent with synovial sarcoma (biphasic type) (23). Fourteen of the 16 tumors displayed histopathological characteristics of $\mathrm{MFH}$ (malignant fibrous histiocytoma, giant cell type) (24-26), with histiocytic and fibroblastic elements arranged in a vague storiform pattern (Fig. 5A). Most of the tumor cells had moderate pleomorphic nuclei. Scattered bizarre cells and a great number of giant cells were noted (Fig. 5B). Immunohistochemical results showed that they were strongly positive for PCNA (Fig. 5C), lysozyme (Fig. 5D) and desmin (Fig. 5E), and partly positive for CD68 (data not shown). No cells reactive to SMA (Fig. 5F), EMA, CD34 and pan-CK were observed in the tumors.

In addition, we isolated 8 monoclonal lines from transformed ADSCs and determined their tumorigenic potential. Each of the 8 monoclonal lines was transplanted into one mouse, and 4 of the 8 clonal lines eventually produced tumors. According to the histologic characteristics and the immunohistochemical data, one tumor was verified to be synovial sarcoma (biphasic type) and one was MFH (giant cell type), while the other two tumors showed a herring bone-like appearance. The cells of these two were characterized by elongated pleomorphic nuclei, though giant cells were not found (Fig. 6A and B). The immunohistochemical results showed that the tumors were strongly positive for vimentin (Fig. 6C) and CD68 (Fig. 6D), revealing them to be fibrosarcoma (27).

\section{Discussion}

There is compelling evidence to support the idea that mesenchymal tumors arise by the transformation of an immature 'fibrohistocytic cell'. This evidence is derived from studies showing that pleomorphic sarcoma cells share their phenotypes with bone marrow-derived mesenchymal progenitor cells (28). These same cells can be converted into Ewing's sarcoma-like cells by the simple transfer of the oncogenic fusion product (EWS-FLI-I) characterizing this specific tumor (18). Although as yet unexplored, a similar result would likely be obtained if analogous oncogenic fusion transcripts (e.g., the liposarcoma FUS-DDIT3 or the synovial 


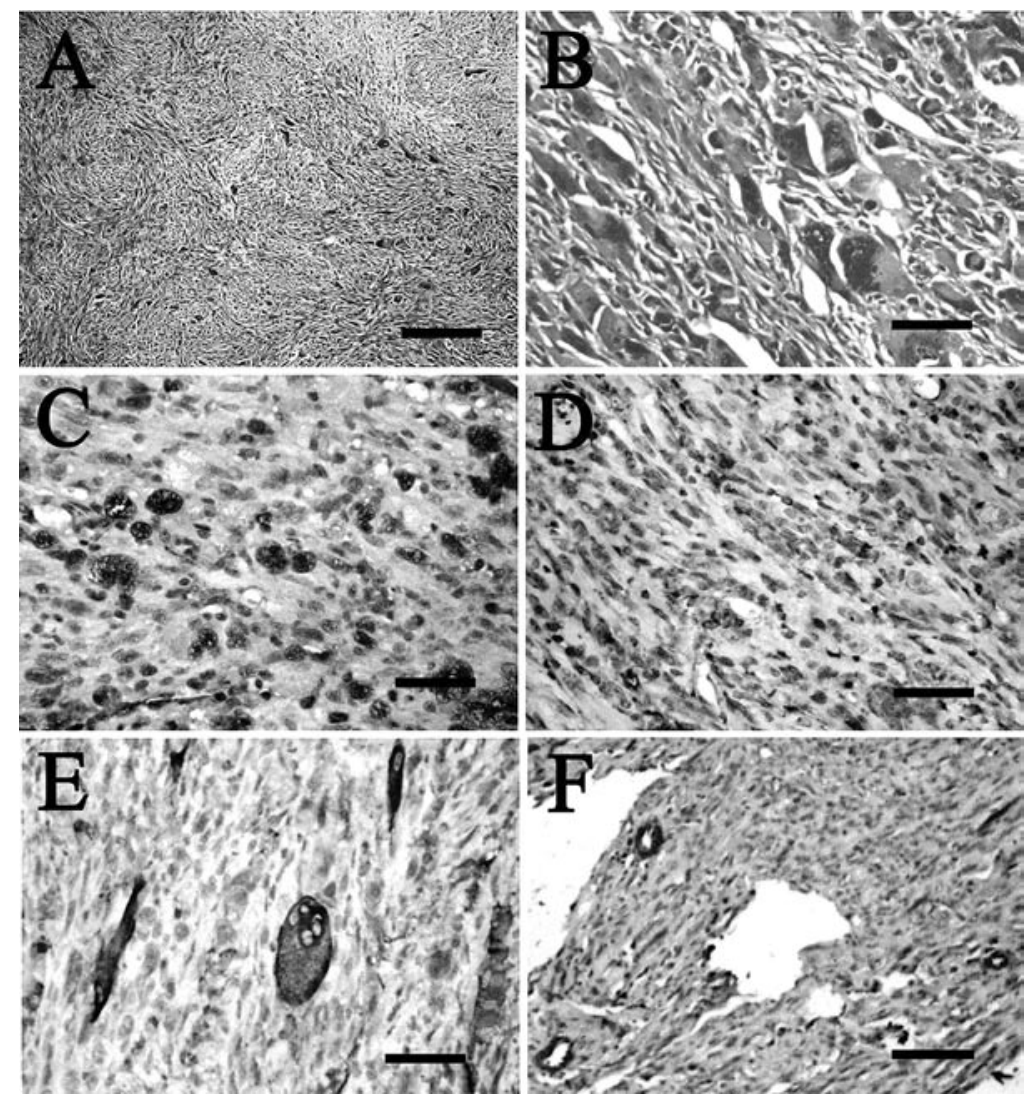

Figure 5. Images of H\&E staining and immunochemical analysis of MFH (giant cell type). (A) Distinctive pleomorphic storiform appearance. (B) A considerable number of generous multinucleated giant cells. Immunochemical images for (C) PCNA, (D) lysozyme and (E) desmin were positive. (F) Immunochemical images for SMA were negative, but normal endothelial cells were positive. Bars: A, $100 \mu \mathrm{m}$; B, C, D and E, $25 \mu \mathrm{m} ; \mathrm{F}, 50 \mu \mathrm{m}$.

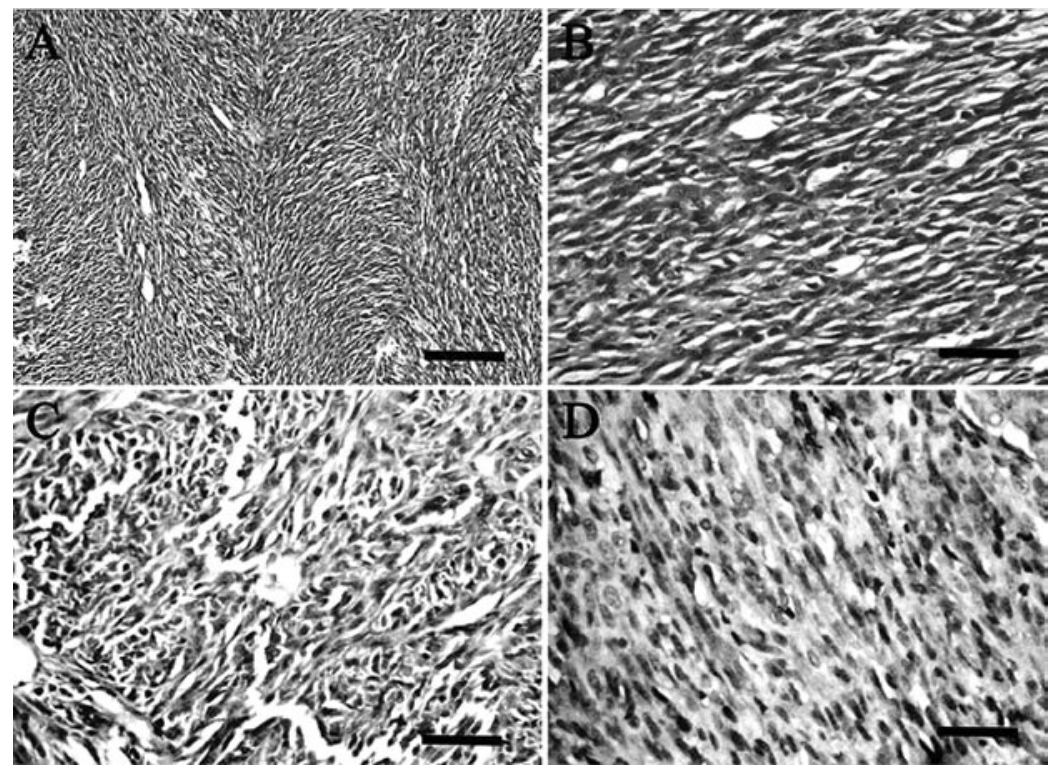

Figure 6. Images of H\&E staining and immunochemical analysis of fibrosarcoma from a monoclone. (A) Herring bone-like appearance of tumor sections. (B) Tumors were composed of interdigitating and interlacing bundles of spindle-shaped fibroblastic cells, which were characterized by elongated pleomorphic nuclei. Immunohistochemical images for (C) vimentin and (D) CD68 were positive. Bars: A, $100 \mu \mathrm{m}$; B, C and D, $25 \mu \mathrm{m}$.

sarcoma SYT-SSX1) were transfected into the above cells. The characteristics of ADSCs are similar to those of bone marrowderived mesenchymal progenitor cells or BMMSCs $(10,11)$, and may be converted into sarcomas by a specific method.
Our study demonstrated that ADSCs are malignantly transformed by MCA induction in vitro. Transformed ADSCs gave rise to different tumor types in vivo when inoculated in nude mice. Therefore, we presume that it is possible for 
multipotent tumor stem cells to exist in transformed ADSCs. This idea is also supported by recent findings regarding the initiation of tumors and the cancer stem cell hypothesis of neoplasias. Studies have confirmed that stem-like cells (tumor stem cells) are present in the pathogenesis of leukemia, breast and brain tumors (29-31). Demonstration of stem-like cells in these tumors provided further support for the stem cell theory of carcinogenesis, from hematologic and ectodermal tumors to mesenchymal tumors (15,29-31). The discovery of multiple types of cancer stem cells suggests that certain tumors containing multiple germ layers may be associated with multiple cancer stem cells. Our study showed that transformed ADSCs express vimentin, the marker for mesoderm, and cytokeratin, the marker for epithelium in vivo; at least two germ layers. Monoclonal transformed ADSCs also produced cells that expressed vimentin and cytokeratin in vivo. These results indicate that transformed ADSCs possess the characteristics of stem cells. Although our study indicates that multipotent cancer stem cells are present in transformed ADSCs, further study is needed to fully comprehend the characteristics of multiple cancer stem cells.

Synovial sarcoma is believed to be a mesenchymal tumor originating from mesenchymal stem cells. However, to date, the exact tumor-initiating cell remains unknown (23). In the present study, we for the first time verified that synovial sarcoma originates from ADSCs. In our experiment, some tumors showed a biphasic, spindle- and epithelial-like appearance, and a gland-like lacune structure formed by epithelial cells. These tumors showed cytoplasmic staining for pan-CK and vimentin, and were also membrane reactive for EMA. It is well known that the diagnosis of synovial sarcoma relies upon histologic and immunohistochemical features. In this case, histologic and immunochemical results were consistent with a finding of synovial sarcoma (23). Therefore, these tumors were considered synovial sarcoma of the biphasic type. In addition, our experimental results showed that synovial sarcoma is derived from transformed ADSCs, indicating a possible cellular origin of synovial sarcoma from ADSCs.

In this study, we provided evidence that ADSCs are the origin of $\mathrm{MFH}$, which is currently considered a high-grade undifferentiated pleomorphic sarcoma, one of the most diffuse and highly aggressive tumors among soft tissue sarcomas in adults. However, the specific cell elements and underlying cell of origin of MFH remain unknown (24-26). Iwasaki et al proposed that MFH may arise from perivascular mesenchymal cells (24). Subsequently, other researchers proposed that MFH is a poorly-differentiated polymorphocellular sarcoma of fibroblastic origin (25). Recently, immortalized human MSCs transfected with SV40-large T antigen were demonstrated to be progenitors of MFH (26). Using a different cell origin and different methods, we confirmed that ADSCs are also tumorinitiating cells of MFH. Considering the similar distribution of STSs and ADSCs in the human body and in our experimental results, we propose that ADSCs are the optimal initiating cell of MFH. Furthermore, we demonstrated that MFH arises from a single clone; it was clear that multiple and polymorphocellular cell elements of MFH have the same and only one progenitor.

To disclose the mystery of the malignant transformation of ADSCs, genetic alterations including abnormalities of the chromosomes and genetic mutations considered to be respon- sible for the development of different types of tumors were examined (32-36). We obtained metaphase chromosomes of transformed ADSCs and found that these cells were aneuploidy, a characteristic of malignant tumors.

The p53 tumor suppressor gene encodes for a multifunctional transcription factor participating in the activation of genes that induce cell cycle arrest, DNA repair and apoptosis following DNA damage, and is the most commonly altered gene in a multitude of human tumors. Alterations of the p53 gene can be acquired somatically or by induction through carcinogens; $74 \%$ of these mutations are missense, resulting in full-length, albeit mutant, proteins. This fraction of missense mutations is much higher than in other tumor suppressor genes, and implies that p53 mutant proteins confer some selective advantage in carcinogenesis (33-36). The vast majority of p53 mutations in human tumors usually occur within four evolutionary conserved domains of the gene located between exons 5 and 8, which contain the sequence-specific DNA binding domain of the protein (34). One research group examined MCA-induced mouse sarcomas for mutations in the p53 gene in one experiment. The rate of mutations of the p53 gene in exons 5-8 was $94 \%(35,36)$. Here, we examined the state of exons 5-9 for p53, and identified point mutations in the p53 gene. We revealed for the first time that the mutation site of exon 5-6 is NT $34907052(\mathrm{~T} \rightarrow \mathrm{G})$; the termination codon changed into serine. Therefore, this alteration of the p53 codon region without a doubt changes the transcription and expression of the p53 protein in transformed ADSCs, and the new gain of function of the mutant p53 protein may play an important role in the malignant transformation process. The mutation site of exon 8-9 was NT $34908189(\mathrm{C} \rightarrow \mathrm{T})$, but mRNA was not expressed in this region, showing it to be a nonsense mutation.

Our research demonstrated that malignant transformed ADSCs induced by MCA in vitro lead to the formation of several types of STSs in vivo, including synovial sarcoma (biphasic type), malignant fibrous histiocytoma (giant cell type) and fibrosarcoma. These findings reveal that many STSs originate from ADSCs. At least one other investigation has convincingly shown that even epithelial tumors (gastric cancers) may arise from cells of a non-epithelial origin (4), and that spontaneously transformed human MSCs lead to carcinoma in vivo and express the marker for epithelium (7). Whether this implies that all epithelial cells have the same formation dynamics remains unknown. Our experiments were similar to the findings of this previous study and extend the concept that tumors originate from cells of other tissue.

In conclusion, we showed that several types of STSs, especially synovial sarcomas, may originate from transformed ADSCs, which are an important initiating cell of various types of STSs.

\section{Acknowledgements}

The authors thank Zheng Shuyan, Su Shaoxue and Liu Junbin for the technical assistance. This work was supported in part by the Natural Science Foundation of China (no. 30672359) (to L.Y.), the Medical Research Foundation of Guangdong Province (no. A2009781) (to L.Y.), and the China Postdoctoral Science Foundation (no. 2004036182) (to L.Y.). 


\section{References}

1. Pardal R, Clarke MF and Morrison SJ: Applying the principles of stem-cell biology to cancer. Nat Rev Cancer 3: 895-902, 2003.

2. Jordan CT: Cancer stem cell biology: from leukemia to solid tumors. Curr Opin Cell Biol 16: 708-712, 2004.

3. Beachy PA, Karhadkar SS and Berman DM: Tissue repair and stem cell renewal in carcinogenesis. Nature 432: 324-331, 2004.

4. Houghton J, Stoicov C, Nomura S, Rogers AB, Carlson J, Li H, Cai X, Fox JG, Goldenring JR and Wang TC: Gastric cancer originating from bone marrow-derived cells. Science 306: $1568-1571,2004$

5. Serakinci N, Guldberg P, Burns JS, Abdallah B, Schrødder H, Jensen T and Kassem M: Adult human mesenchymal stem cell as a target for neoplastic transformation. Oncogene 23: 5095-5098, 2004.

6. Rubio D, Garcia-Castro J, Martín MC, de la Fuente R, Cigudosa JC, Lloyd AC and Bernad A: Spontaneous human adult stem cell transformation. Cancer Res 65: 3035-3039, 2005

7. Rubio D, Garcia S, de la Cueva T, Paz MF, Lloyd AC, Bernad A and Garcia-Castro J: Human mesenchymal stem cell transformation is associated with a mesenchymal-epithelial transition. Exp Cell Res 314: 691-698, 2008

8. Meyerrose TE, de Ugarte DA, Hofling AA, Herrbrich PE, Cordonnier TD, Shultz LD, Eagon JC, Wirthlin L, Sands MS Hedrick MA and Nolta JA: In vivo distribution of human adipose-derived mesenchymal stem cells in novel xenotransplantation models. Stem Cells 25: 220-227, 2007.

9. Sonoda E, Aoki S, Uchihashi K, Soejima H, Kanaji S, Izuhara K, Satoh S, Fujitani N, Sugihara H and Toda S: A new organotypic culture of adipose tissue fragments maintains viable mature adipocytes for a long term, together with development of immature adipocytes and mesenchymal stem cell-like cells Endocrinology 149: 4794-4798, 2008

10. Zuk PA, Zhu M, Mizuno H, Huang J, Futrell JW, Katz AJ, Benhaim P, Lorenz HP and Hedrick MH: Multilineage cells from human adipose tissue: implications for cell-based therapies. Tissue Eng 7: 211-228, 2001.

11. Zuk PA, Zhu M, Ashjian P, de Ugarte DA, Huang JI, Mizuno H, Alfonso ZC, Fraser JK, Benhaim P and Hedrick MH: Human adipose tissue is a source of multipotent stem cells. Mol Biol Cell 13: 4279-4295, 2002

12. Kotilingam D, Lev DC, Lazar AJ and Pollock RE: Staging soft tissue sarcoma: evolution and change. CA Cancer J Clin 56 282-291, 2006

13. Spunt SL, Skapek SX and Coffin CM: Pediatric nonrhabdomyosarcoma soft tissue sarcomas. Oncologist 13: 668-678, 2008.

14. Tolar J, Nauta AJ, Osborn MJ, et al: Sarcoma derived from cultured mesenchymal stem cells. Stem Cells 25: 371-379, 2007.

15. Liu C, Chen Z, Chen Z, Zhang T and Lu Y: Multiple tumor types may originate from bone marrow-derived cells. Neoplasia 8 : 716-724, 2006

16. Zhou YF, Bosch-Marce M, Okuyama H, Krishnamachary B, Kimura H, Zhang L, Huso DL and Semenza GL: Spontaneous transformation of cultured mouse bone marrow-derived stromal cells. Cancer Res 66: 10849-10854, 2006.

17. Miura M, Miura Y, Padilla-Nash HM, Molinolo AA, Fu B, Patel V, Seo BM, Sonoyama W, Zheng JJ, Baker CC, Chen W, Ried T and Shi S: Accumulated chromosomal instability in murine bone marrow mesenchymal stem cells leads to malignant transformation. Stem Cells 24: 1095-1103, 2006.
18. Riggi N, Cironi L, Provero P, Suvà ML, Kaloulis K, GarciaEcheverria C, Hoffmann F, Trumpp A and Stamenkovic I: Development of Ewing's sarcoma from primary bone marrowderived mesenchymal progenitor cells. Cancer Res 65: 11459-11468, 2005.

19. Bottone MG, Malgara R, Pellicciari C and Fuhrman Conti AM: Treatments with saline solutions and DNase I have different effects on DNA content and distribution in human and in mouse chromosomes. Eur J Histochem 40: 101-108, 1996.

20. Yang LY, Liu XM, Sun B, Hui GZ, Fei J and Guo LH: Adipose tissue-derived stromal cells express the phenotypes of neurons. Chin Med J 117: 425-429, 2004.

21. Yang L, Chen Q, Zhang S, Wang X, Li W, Wen J, Huang X, Zheng J, Huang G, Huang T and Ju G: A novel mutated cell line with characteristics of dedifferentiated chondrosarcoma. Int $J$ Mol Med 24: 427-35, 2009.

22. Woodbury D, Schwarz EJ, Prockop DJ and Black IB: Adult rat and human bone marrow stromal cells differentiate into neurons. J Neurosci Res 61: 364-370, 2000.

23. Scheithauer BW, Silva AI, Kattner K, Seibly J, Oliveira AM and Kovacs K: Synovial sarcoma of the sellar region. Neuro Oncol 9: 454-459, 2007.

24. Iwasaki H, Isayama T, Johzaki $H$ and Kikuchi M: Malignant fibrous histiocytoma. Evidence of perivascular mesenchymal cell origin immunocytochemical studies with monoclonal anti-MFH antibodies. Am J Pathol 128: 528-537, 1987.

25. Pozharisskiú KM and Nikitin AI: The morphogenesis and histogenesis of an experimental malignant fibrous histiocytoma. Vopr Onkol 37: 51-59, 1991.

26. Matushansky I, Hernando E, Socci ND, Mills JE, Matos TA, Edgar MA, Singer S, Maki RG and Cordon-Cardo C: Derivation of sarcomas from mesenchymal stem cells via inactivation of the Wnt pathway. J Clin Invest 117: 3248-3257, 2007.

27. Scott SM, Reiman HM, Pritchard DJ and Ilstrup DM: Soft tissue fibrosarcoma. A clinicopathologic study of 132 cases. Cancer 64: 925-931, 1989

28. Gazziola C, Cordani N, Wasserman B, Carta S, Colombatti $\mathrm{A}$ and Perris R: Malignant fibrous histiocytoma: a proposed cellular origin and identification of its characterizing gene transcripts. Int J Oncol 23: 343-351, 2003

29. Bonnet D and Dick JE: Human acute myeloid leukemia is organized as a hierarchy that originates from a primitive hematopoietic cell. Nat Med 3: 730-737, 1997.

30. Hemmati HD, Nakano I, Lazareff JA, Masterman-Smith M, Geschwind DH, Bronner-Fraser M and Kornblum HI: Cancerous stem cells can arise from pediatric brain tumors. Proc Natl Acad Sci USA 100: 15178-15183, 2003.

31. Singh SK, Clarke ID, Terasaki M, Bonn VE, Hawkins C, Squire J and Dirks PB: Identification of a cancer stem cell in human brain tumors. Cancer Res 63: 5821-5828, 2003.

32. Lengauer C, Kinzler KW and Vogelstein B: Genetic instabilities in human cancers. Nature 396: 643-649, 1998.

33. Hung J and Anderson R: p53: functions, mutations and sarcomas. Acta Orthop Scand Suppl 273: 68-73, 1997.

34. Andreassen A, Oyjord T, Hovig E, et al: p53 abnormalities in different subtypes of human sarcomas. Cancer Res 53: 468-471, 1993.

35. Shimokado K, Watanabe H, Sumii M, Miyagawa K, Kamiya K, Dohi K and Niwa O: p53 gene mutation and loss of heterozygosity of chromosome 11 in methylcholanthrene-induced mouse sarcomas. Jpn J Cancer Res 89: 269-277, 1998.

36. Watanabe $\mathrm{H}$, Shimokado $\mathrm{K}$, Asahara T, Dohi $\mathrm{K}$ and Niwa $\mathrm{O}$ : Analysis of the c-myc, K-ras and p53 genes in methylcholanthrene-induced mouse sarcomas. Jpn J Cancer Res 90: 40-47, 1999. 